\title{
Uma Abordagem Visual para Evolução de Test Smells em Sistemas de Software Java
}

\author{
Adriana P. S. Cruz ${ }^{1}$ (mestrando), Heitor Costa (orientador) ${ }^{1}$ \\ ${ }^{1}$ Mestrado Acadêmico em Ciência da Computação \\ Programa de Pós-Graduação em Ciência da Computação (PPGCC) \\ Departamento de Ciência da Computação - Universidade Federal de Lavras (UFLA) \\ Câmpus Universitário - Aquenta Sol - CEP 37200-000 Lavras - MG - Brazil \\ Ingresso: 08/2019 - Qualificação: 08/2020 - Previsão Defesa: 07/2021 \\ adriana.cruz@estudante.ufla.br, heitordufla.br
}

Resumo. O teste de software é parte do desenvolvimento de software. Durante o teste, podem surgir más escolhas de implementação do código de teste, levando a inserção de Test Smells. A presença de Test Smells em código de teste pode o tornar ineficaz em encontrar bugs, gerando retrabalho e custos adicionais. A manutenção do código de teste ocorre por causa da evolução do código de produção. Para visualizar a evolução de sistemas de software, têm sido utilizadas técnicas de visualização de software. Apesar dessas técnicas contribuírem para visualizar a evolução de sistemas de software, elas não abrangem código de teste para visualização de Test Smells. Dessa forma, no trabalho de mestrado, o objetivo é proporcionar melhor entendimento das ocorrências de Test Smells e sua evolução em código de teste aos Gerentes de Testes/Testadores, para agilizar a identificação de problemas nos casos de testes, melhorando sua qualidade. Para isso, será elaborada uma abordagem para visualizar essa evolução.

Palavras-Chave. Evolução de Software, Visualização de Software, Test Smells.

Evento CBSoft: SBES 


\section{Caracterização do Problema}

Teste de software é o processo de executar o software de maneira controlada para verificar se o software se comporta conforme o especificado, sendo fundamental para a sua avaliação durante seu desenvolvimento [Crespo et al., 2004]. O teste não garante um software livre de erros, porém é necessário ter testes durante o projeto de sistemas de software, para minimizar os erros e diminuir custos [Silva et al., 2016]. Por causa da quantidade de código de teste que as empresas lidam, há a preocupação com a qualidade desses códigos, surgindo a Engenharia de Código de Teste de Software (ECTS) (Software Test-Code Engineering), que refere-se aos métodos, às técnicas e às ferramentas utilizadas para verificar e manter código de teste [Yusifoğlu et al., 2015]. Na prática, nem sempre a ECTS é seguida, resultando na inserção de anti-padrões em códigos de testes (Test Smells) [Palomba et al., 2018]. Test Smells (TS) são más escolhas de design/implementação do código de testes e sua presença pode afetar negativamente a compreensão e a manutenção desse código. Eles podem ocasionar testes menos eficazes em encontrar bugs no código de produção [Spadini et al., 2018]. Diante disso, algumas ferramentas foram propostas para automatizar a detecção de $T S$. Dentre essas ferramentas, destacam-se JNose Test [Vírginio et al., 2019], que detecta 21 tipos de $T S$ e a quantidade de vezes em que eles ocorrem e a ferramenta VITrUM [Pecorelli et al., 2020], que analisa classes de teste e utiliza cores diferentes conforme a presença de $T S$, sendo possível filtrar os resultados por período e por tipo de $T S$.

Assim como o código de produção passa por evoluções, o código de teste evolui para atender novos casos de teste. Não se sabe ao certo se a evolução do código de teste é responsável por introduzir TS [Greiler et al., 2013], mas, mesmo que eles sejam removidos, outros podem ser inseridos [Kim, 2019]. Técnicas de visualização de software têm sido utilizadas para mostrar a evolução de sistemas de software para facilitar a sua compreensão [Bastos; Costa, 2016]. Apesar disso, elas não abrangem a evolução de códigos de teste para visualização de $T S$. Desse modo, não foram encontradas na literatura ferramentas que utilizam técnicas de visualização de software para mostrar a presença de $T S$ em códigos de teste durante sua evolução [Garousi et al., 2018]. Dessa forma, nesse trabalho de mestrado, o objetivo é proporcionar melhor entendimento da evolução de $T S$ em código de teste aos Gerentes de Testes/Testadores. Para isso, será elaborada uma abordagem para visualizar essa evolução para facilitar o processo de identificação de problemas no código de teste e melhorar sua qualidade. Esse trabalho está no escopo de um framework conceitual em desenvolvimento por um grupo de pesquisadores interinstitucional, que consiste na integração de componentes para prevenção, identificação e correção de $T S$ para melhorar a qualidade de código de teste.

Este trabalho está organizado da seguinte forma. Fundamentação teórica é apresentada na Seção 2. O método de pesquisa para a realização do trabalho é detalhado na Seção 3. Avaliação dos resultados é discutida na Seção 4. Contribuições esperadas são apresentadas na Seção 5. Trabalhos relacionados são apresentados na Seção 6. 


\section{Fundamentação Teórica}

\subsection{Test Smells}

Para minimizar erros no código de produção, esforços têm sido voltados para o desenvolvimento de código de testes com mais qualidade [Tufano et al., 2016]. Contudo, a presença de $T S$ tem impacto negativo sobre manutenção do código de teste por gerarem testes complexos e difíceis de entender e modificar, prejudicando a independência e a estabilidade dos testes [Van Rompaey, 2006]. Como indicadores de possíveis TS, tem-se a maneira como os casos de teste são documentados ou organizados e como os casos de teste interagem com outros testes, com o código de produção e com recursos externos [Tufano et al., 2016]. Muitas vezes, TS não são percebidos pelos testadores como problemas reais de design. Além disso, o responsável pela inserção do $T S$ pode não identificar a sua presença em seu próprio código [Spadini et al., 2018]. Contudo, estudos destacaram empiricamente o efeito negativo de TS quanto à compreensibilidade e à manutenibilidade do código, destacando a importância de investir esforços no desenvolvimento de ferramentas para TS [Tufano et al., 2016].

$\mathrm{Na}$ literatura, foi introduzido um conjunto com 30 tipos de TS [Van Deursen et al., 2001; Meszaros, 2006; Peruma, 2019]. Desse conjunto, foram selecionados 21 TS para serem investigados durante a evolução do código de teste apresentada neste trabalho. Esses TS foram escolhidos por terem sido formalmente validados em uma pesquisa com desenvolvedores [Peruma, 2019]. Além disso, 21 TS são detectados pela ferramenta utilizada neste trabalho (JNose Test [Virgínio et al., 2019]).

\subsection{Visualização de Software}

Visualização é o processo de transformar informações em uma forma visual, permitindo aos usuários observar a informação. Visualização de software tem sido utilizada na Engenharia de Software para facilitar a tarefa de compreensão, de manutenção e de evolução de sistemas de software [Diehl, 2007; Conceição et al., 2012]. Ao utilizar recursos gráficos, a compreensão torna-se mais intuitiva do que representações textuais. Ao representar um sistema de software por meio de recursos visuais, a sua representação mental pode ser clara, pois os seres humanos possuem mais facilidade em entender informações representadas de forma gráfica [Caserta; Zendra, 2011]. Em um estudo [Cruz et al., 2016], 20 técnicas de visualização de software foram identificadas.

Um sistema de software deve ser constantemente mantido. Para isso, evoluções no código de produção são necessárias para atender novos requisitos, corrigir bugs/design ou melhorar o desempenho [Sulaiman; 2004], o que acarreta na evolução do código de teste. A compreensão dessas evoluções pode ser usada para encontrar a origem de problemas atuais ou obter informações que possibilitam prever características futuras desses sistemas. No entanto, o aumento da quantidade de informações, funções e quantidade de código pode deixar o sistema mais complexo, dificultando sua compreensão. Desse modo, técnicas de visualização de software têm sido usadas para representar essas evoluções [Bastos; Costa, 2016].

Para a realização do trabalho de mestrado será utilizada as técnicas Graphs (Grafos) e Timeline. Graphs é utilizada para a representação estrutural dos dados, sendo capaz de mostrar a ligação entre os artefatos de software [Herman et al., 2000].. Timeline é utilizada como estratégia de análise da evolução de software, retratando a 
evolução do projeto de software considerando várias versões disponíveis para análise. Dadas 2 versões de um projeto de software ( $\mathrm{v}_{1}$ e $\left.\mathrm{v}_{2}\right)$, essa análise permite a comparação dessas versões sendo possível analisar as mudanças entre diferentes versões sobre o ciclo de evolução do software. Além disso, será utilizada a perspectiva de autoria para identificar quem possivelmente inclui (assumiu a autoria de) TS, ela exibe as atividades dos autores durante a evolução do software (e.g., quantos commits executaram ou em quais arquivos eles trabalharam [Novais et al., 2013]).

\section{Método de Pesquisa}

O método de pesquisa (Figura 1) a ser utilizado para o desenvolvimento da pesquisa de Mestrado possui quatro etapas:

- Etapa 1 - Revisão da Literatura (Estado: Concluído). Nessa etapa, foram buscados na literatura artigos sobre $T S$, técnicas de visualização de software para $T S$, evolução de $T S$ e identificação de autores de $T S$. Essa busca foi realizada nas bases de bibliotecas científicas virtuais, por serem amplamente utilizadas no meio acadêmico: i) IEEE Xplore (http://ieeexplore.ieee.org); ii) ACM Digital Library (http://dl.acm.org); iii) Scopus (http://www.scopus.com); iv) Ei Compendex (http://www.engineeringvillage.com/); e v) Google Scholar (https://scholar.google.com.br/);

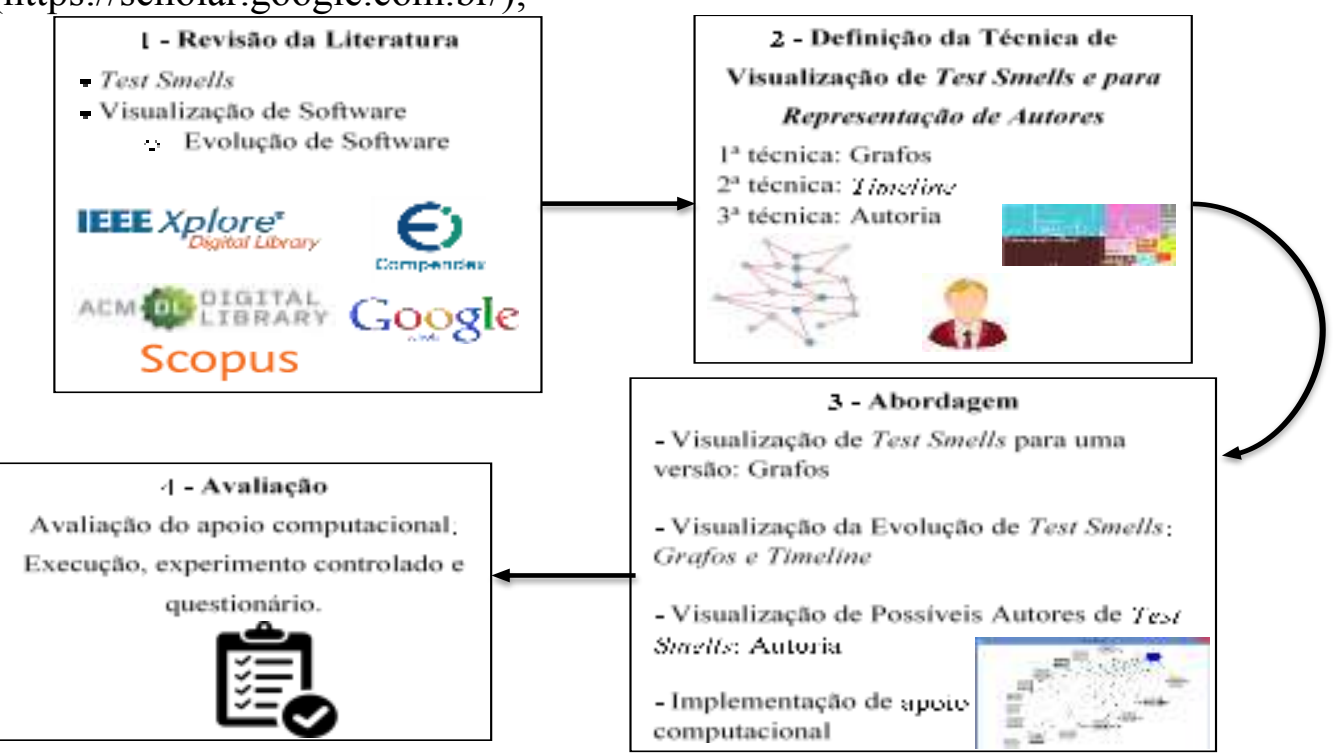

Figura 1. Método da Pesquisa

- Etapa 2 - Definição de Técnicas para a Visualização de TS (Estado: Concluído). Nessa fase, foi definida perante as técnicas identificadas, as técnicas mais adequadas ao propósito de exibir visualmente os TS. Primeiramente, para a visualização das ocorrências de $T S$ foi escolhida a técnica de Graph por ser capaz de mostrar a ligação entre os artefatos de software. Assim, pudemos representar os TS e suas relações com o projeto, as classes de teste e com autores. Para a visualização da evolução de TS (duas versões) foi utilizada também a técnica de Graph e foi definida mais uma a técnica denominada Timeline que é mais intuitiva para exibir a evolução, pois fornece visualmente um comparativo. Os possíveis autores são representados nos grafos; 
- Etapa 3 - Abordagem (Estado: Em andamento). Nessa fase, há a elaboração da abordagem e a implementação da visualização das ocorrências e evolução de $T S$, e seus possíveis autores. Até o momento, foi implementada as duas técnicas de visualização definidas para até duas versões de sistema de software java, foi definida duas versões, para gerar uma visualização mais clara e menos sobrecarregada. A abordagem recebe como entrada o arquivo .csv gerado pela ferramenta JNose. O usuário pode selecionar entre 5 granularidades: Project, All Test Classes, A Specific Test Class, A Specific Test Smells e Author. Pretende-se incluir os métodos que contém os TS em ambas visualizações. Na Figura 2 podemos ver a visualização da técnica Graph a esquerda, onde é fornecido todos tipos de $T S$ da classe de teste em questão, nas arestas é informado as ocorrências. Podemos ver que na versão 2 a classe de teste foi removida (ícone x). O sufixo (_1 ou _2) representa a ordem de inserção das versões. No lado direito da figura 2, é fornecida a técnica de visualização Timeline onde é apresentado por classes os TS existentes, ao passar o mouse sobre as componentes informações como nome e ocorrências são fornecidas, as cores representam o comportamento do $T S$ durante a evolução.

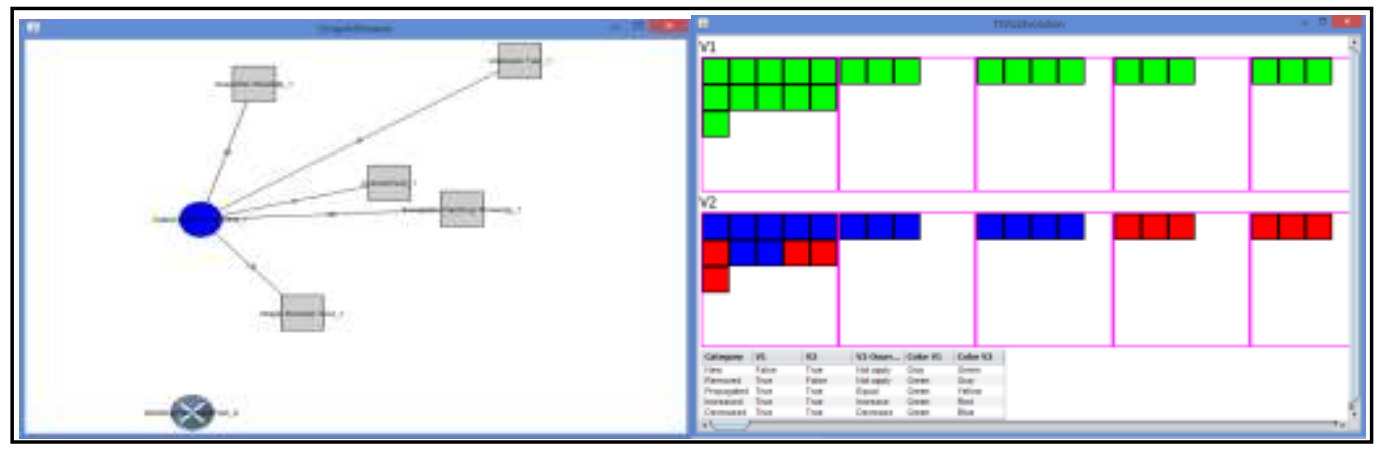

Figura 2. Visualização Graph View e Timeline View - Commons $10^{1}$

- Etapa 4 - Avaliação (Estado: Não iniciado). Após a finalização, a ferramenta computacional, que automatiza a abordagem proposta, será avaliada, a fim de identificar se facilitou o processo de identificação/visualização dos TS perante a evolução do código de teste. Para isso, será executada a ferramenta em diversas sistemas de software (quantitativamente) e será realizado um experimento controlado com a aplicação de um questionário para os convidados apresentarem sua percepção sobre a ferramenta proposta (qualitativamente).

\section{Avaliação dos Resultados}

A abordagem será avaliada de forma quantitativa e qualitativa. A avaliação quantitativa será através da execução da ferramenta em diversas versões de diversos sistemas de software disponíveis no GitHub ${ }^{2}$ utilizados em um trabalho anterior para a detecção de $T S$ [Virgínio et al., 2019], para verificar o comportamento dos $T S$ durante a evolução do código de teste. $\mathrm{E}$ a avaliação qualitativa será através da execução de um experimento controlado com 16 participantes (múltiplo de 4 conforme design Quadrado latino) e 3 ferramentas (TestQ, VITRuM e ferramenta proposta) para verificar se a abordagem facilitou, tornou mais rápida a tarefa de identificar/visualizar problemas durante a

\footnotetext{
${ }^{1} \mathrm{https}$ //commons.apache.org/proper/commons-io/ 
evolução do código de teste e com a aplicação de questionário para coletar pontos positivos, negativos e melhorias para a ferramenta proposta.

\section{Contribuições Esperadas}

A abordagem desenvolvida será avaliada com a utilização quanto a sua eficácia em atender ao propósito para identificar se realmente facilita visualizar/acompanhar ocorrências de $T S$ nos códigos de testes. O objetivo é verificar se a abordagem proposta pode apoiar os Gerentes de Testes/Testadores no processo de tomada de decisão e se facilita encontrar problemas no código de teste. Com isso, pretende-se responder as seguintes questões: i) "Qual é tempo para visualizar $T S$ em várias versões de um sistema de software?" ii) "A identificação dos possíveis autores facilitou o processo de tomada de decisão ao Gerentes de Testes/Testadores?", iii) "Visualização de ocorrências de TS no código de teste foi intuitiva para Gerentes de Testes/Testadores?" e iv) "Durante a evolução do código de teste os TS diminuíram ou aumentaram?".

\section{Trabalhos Relacionados}

A ferramenta TestQ [Breugelmans; Van Rompaey, 2008] suporta várias maneiras de visualizar dados para explorar a estrutura do código de teste e quantificar a presença de 12 tipos de TS. Essa ferramenta apresenta a visualização Test Suite Topology, representando os dados da estrutura da suíte de testes em uma árvore expansível. A ferramenta também apresenta a visualização Smell Flower que permite ao usuário explorar pontos de TS (hotspots) por meio de sua representação em gráficos e quantificar instâncias individuais usando um gráfico de pizza.

A ferramenta VITrUM [Pecorelli et al., 2020] inclui o cálculo de medidas de código de teste relacionadas à coesão, ao acoplamento e à complexidade, detecta 7 tipos de $T S$ e calcula a cobertura das classes de teste. Como resultado, ela apresenta uma lista de classes de teste em três cores (vermelho: classes de teste afetadas por $T S$ com valores de medidas de código de teste acima dos limites definidos, amarelo: classes de teste afetadas por $T S$ com valores de medidas de código de teste nos limites definidos e cinza: classes de teste sem TS). Além disso, o usuário pode filtrar os resultados por tipo de $T S$ para exibir sua evolução em um gráfico ao longo do desenvolvimento do projeto.

Diferentemente das ferramentas apresentadas, a ferramenta proposta se concentra em mostrar a ocorrência de 21 tipos de $T S$ por meio de duas técnicas de visualização (Grafos e Timeline), apresentando suas relações de projeto, classes, e possíveis autores. As ferramentas encontradas não abrangem quantidade maior de $T S$ associados às técnicas de visualização de software nem apresentou a comparação durante a evolução das versões e possíveis autores (autoria).

\section{Referências}

Bastos, C.; Costa, H. (2016) "Uma Abordagem para Visualização da Evolução de Código Morto em Sistemas de Software Orientados a Objetos". In: Workshop de Teses e Dissertações em Qualidade de Software, p.33.

Breugelmans, M.; Van Rompaey, B. (2008) "Testq: Exploring Structural and Maintenance Characteristics of Unit Test Suites" In: International Workshop on Advanced Software Development Tools and Techniques.

Caserta, P.; Zendra, O. (2011) "Visualization of the Static Aspects of Software: A Survey". In: Visualization and Computer Graphics, v. 17, n. 7, p. 913-933. 
Conceição, C. F. R; Carneiro, G.; David, J. M. N. (2012) "Usando Recursos de Visualização Enriquecidos com Elementos de Percepção para a Compreensão de Software em um Ambiente de Desenvolvimento Distribuído”. In: WBVS. p. 41-48.

Crespo, A. N., Silva, O. J., Borges, C. A., Salviano, C. F., Argollo, M., Jino, M. (2004) "Uma Metodologia para Teste de Software no Contexto da Melhoria de Processo". In: Simpósio Brasileiro de Qualidade de Software, p. 271-285.

Cruz, A., Bastos, C., Afonso, P., Costa, H. (2016) "Software Visualization Tools and Techniques: A Systematic Review of the Literature". In: SCCC. p. 1-12.

Diehl, S. (2007) "Software Visualization: Visualizing the Structure, Behaviour, and Evolution of Software", Berlim: Springer. 186p.

Garousi, V., Kucuk, B., Felderer, M. (2018) "What We Know About Smells in Software Test Code”. In: IEEE Software. p. 61-73.

Greiler, M.; Van Deursen, A.; Storey, M. A. (2013) "Automated Detection of Test Fixture Strategies and Smells”. In: ICST. p. 322-331.

Herman, I., G. Melançon, and M. S. Marshall (2000). "Graph visualization and navigation in information visualization: a survey". In: IEEE Transactions on Visualization and Computer Graphics. p. 24-43.

Kim, D. J. (2019) "An Empirical Study on the Evolution of Test Smell", https://djaekim.github.io/djae.io/img/EvolutionOfTestSmell.pdf. Acesso: 02/04/2020.

Novais, L. R.; Nunes, C.; Garcia, A.; Mendonça, M. Sourceminer Evolution: A Tool for Supporting Feature Evolution Comprehension. In: ICSM. p. 508-511, 2013.

Palomba, F.; Zaidman, A.; De Lucia, A. (2018) “Automatic Test Smell Detection Using Information Retrieval Techniques”. In: ICSME. p. 311-322.

Pecorelli, F.; do Lillo, G.; Palomba, F.; de Lucia, A. (2020) "VITRuM-A Plug-in for the Visualization of Test-Related Metrics." International Conference on Advanced Visual Interfaces, 1-3.

Peruma, A.; Almalki, K.; Newman, C. D.; Mkaouer, M. W.; Ouni, A.; Palomba, F. (2019) "On the Distribution of Test Smells in Open Source Android Applications: An Exploratory Study". In: International Conference on Computer Science and Software Engineering. p. 193-202.

Silva, R. O.; Machado, G. B. G.; Viana, G. B.; dos Santos Silva, J. S. (2016) "O Processo de Teste de Software”. In: Tecnologias em Projeção. v. 7.

Spadini, D.; Palomba, F.; Zaidman, A.; Bruntink, M.; Bacchelli, A. (2018) "On the Relation of Test Smells to Software Code Quality". In: ICSME. p. 1-12.

Sulaiman, S. (2004) "Viewing Software Artifacts for Different Software Maintenance Categories Using Graph Representations". In: Journal of Science. v. 17, p. 55-67.

Tufano, M.; Palomba, F.; Bavota, G.; Di Penta, M.; Oliveto, R.; De Lucia, A.; Poshyvanyk, D. (2016) "An Empirical Investigation into the Nature of Test Smells". In: International Conference on Automated Software Engineering, p. 4-15.

Van Deursen, A.; Moonen, L.; Van Den Bergh, A.; Kok, G. (2001) "Refactoring Test Code". In Conference on extreme programming and flexible processes in software engineering. p. 92-95.

Van Rompaey, B., Du Bois, B., Demeyer, S. (2006) "Characterizing the Relative Significance of a Test Smell". In: ICSM p. 391-400.

Virgínio, T.; Santana, R.; Martins, L. A.; Soares, L. R.; Costa, H.; Machado, I. (2019) "On the Influence of Test Smells on Test Coverage". In: SBES. p. 467-471.

Yusifoğlu, V. G.; Amannejad, Y.; Can, A. B. (2015) "Software Test-Code Engineering: A Systematic Mapping”. In: Information and Software Technology. p. 123-147. 